\title{
Combination of time dilation and gravitational time dilation
}

\author{
Andrea Conte ${ }^{1, *}$
}

\begin{abstract}
In compliance with the principle of relativity, a time dilation equation expressed as an energy ratio is used to combine time dilation due to motion and due to gravitational attraction. To show the correlation with the time dilation equations, the Lorentz factor and the gravitational time dilation equations are derived from the equation. The equivalence between the time dilation due to motion and due to gravitational attraction emerges and a combination of both is made possible using the energy ratio equation.

Keywords: Time dilation, Energy ratio, Mass, Drag force, Special Relativity, Relativity, Lorentz Factor, Equivalence principle, Gravitational time dilation, Time dilation combination
\end{abstract}

\footnotetext{
*Corresponding author

Email address: conte.an@hotmail.com (Andrea Conte)

${ }^{1}$ Declarations of interest: none
} 


\section{Contents}

1 Introduction 3

2 Inertia property of mass as a drag force 3

3 Time dilation as an energy ratio 4

3.1 Action due to motion . . . . . . . . . . . . . . . . . 4

3.2 Action due to gravitational attraction . . . . . . . . . . . . 5

4 Combination of motion and gravitational time dilation 6

4.1 Using the standard equations . . . . . . . . . . . . . . . . . . . . . . . . . . . .

4.2 Using the energy ratio equation . . . . . . . . . . . . . . . . 7

$\begin{array}{llr}5 & \text { Conclusion } & 7\end{array}$

$\begin{array}{llll}\text { Appendix A } & \text { Supplementary data } & 7\end{array}$ 


\section{Introduction}

Before presenting the equation that combines time dilation and gravitational time dilation, an introduction is made concerning the value $m v^{2}$ which is used in that equation. This value is interpreted as representing a resisting effect encountered during motion. This effect is more commonly named a drag force. A drag force is the resistance against an object in motion provoked by the fluid in which the object travels. Taking a Newtonian perspective, the aether $[7,10]$ would be the medium that provides a drag force required to provoke a time dilation of an object in motion. However, this idea would put at risk the basic principle of relativity $[4,5,6]$ which indicates that no medium is present to provoke a time dilation. An alternative solution to the absence of a medium is the idea that the effect of a drag force might not be provoked by an outside force but might be related to the properties of an object mass. Indeed, the mass exerts a resisting force when an external force is applied to it. This resisting property is called the inertia property of a mass. When a force is applied to put a mass into motion, the mass itself acts as a resisting force from the point of view of the applied force. In this scenario, the mass behaves like a drag force [8]. The time dilation due to motion presented in Eq.(8) is calculated by subtracting a $m v^{2}$ value suggesting a drag force effect. Choosing the inertia property of the mass playing this resisting role in time dilation should not contradict the principle of relativity. Before presenting the energy ratio equation and the combination of time dilation equations in section 3 and 4 respectively, the next section demonstrates the hypothetical similitude between $m v^{2}$ and the work done by a drag force called drag energy.

\section{Inertia property of mass as a drag force}

This sections shows that $m v^{2}$ can be made equivalent to a drag energy by deriving it from the standard drag force formula. In fluid dynamics, a drag force is proportional to $v^{2}$. Its formula is represented by the following equation.

$$
\text { Drag Force }=\left(\frac{1}{2} \rho C_{D} A\right) v^{2}
$$

Drag Force $\propto v^{2}$

Eq.(2) shows that the drag force is proportional to $v^{2}$. By multiplying $v^{2}$ by a mass in Eq.(2), a drag energy value can be obtained.

$$
\text { Drag Energy } \propto(m) v^{2}
$$

To derive Eq.(3) from Eq.(1), a length is added in both sides of Eq.(1) to express the work done by the drag force. This work represents the drag energy.

$$
\text { Drag Force } * L=\left(\frac{1}{2} \rho C_{D} A * L\right) v^{2}
$$


Both sides of Eq.(4) express an energy value. The following equivalence can then be done between Eq.(3) and Eq.(4).

$$
\text { (m) } v^{2}=\left(\frac{1}{2} \rho C_{D} A * L\right) v^{2}
$$

To find the drag coefficient $C_{D}$, all other variables are set to 1 . After solving the equivalence, the drag coefficient $C_{D}$ will become 2 which verifies the equivalence. This value of $m v^{2}$ which is considered to be equivalent to a drag energy will be used in section 3 in Eq.(8) to calculate the time dilation due to motion.

\section{Time dilation as an energy ratio}

The equation presented in this section was used to build a model on time perception [2]. This section focuses only on the energy ratio to calculate time dilation. Simple derivations will be done to demonstrate the correlation with the Lorentz factor and the gravitational time dilation equation. The energy ratio equation is expressed by the following equation.

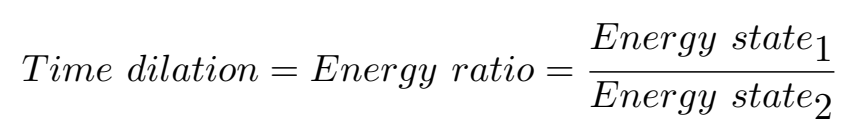

The energy ratio equation compares two states of a system. At rest, the system has an internal energy of $m c^{2}$ [3]. The ratio is shown is Eq.(7). The method to obtain this ratio and the reason to add a square root around it are explained in [2].

$$
\text { Ratio }=1=\frac{\text { State }_{1}}{\text { State }_{2}}=\sqrt{\frac{m c^{2}}{m c^{2}}}
$$

A ratio of 1 means no dilation is present between the two states. To obtain a dilation, an external action on the system is needed. Two actions are presented, an action due to motion and the other due to gravitational attraction.

\subsection{Action due to motion}

The action due to motion is represented by subtracting $m v^{2}$.

$$
\text { Ratio }=\frac{\text { State }_{1}}{\text { State }_{2}}=\sqrt{\frac{m c^{2}-m v_{1}^{2}}{m c^{2}-m v_{2}^{2}}}
$$

If $m v_{1}$ is greater than $m v_{2}$, the ratio will be less than 1 . This ratio gives a time dilation between two states. When $v_{2}=0$ and $v_{1}>0$, this ratio gives the same result as the Lorentz factor denominator. Inverting this ratio, meaning dividing the value 1 by this ratio, gives the same result as the Lorentz factor in its standard form. The next equation shows the 
derivation to obtain the Lorentz factor denominator when $v_{2}=0$ and $v_{1}>0$.

$$
\begin{aligned}
\frac{\text { State }_{1}}{\text { State }_{2}} & =\sqrt{\frac{m c^{2}-m v_{1}^{2}}{m c^{2}-m v_{2}^{2}}}=\sqrt{\frac{m c^{2}-m v_{1}^{2}}{m c^{2}-0}}=\sqrt{\frac{m c^{2}-m v_{1}^{2}}{m c^{2}}} \\
& =\sqrt{\frac{c^{2}}{c^{2}}-\frac{v_{1}^{2}}{c^{2}}}=\sqrt{1-\frac{v_{1}^{2}}{c^{2}}}=\text { Lorentz factor denominator }
\end{aligned}
$$

\subsection{Action due to gravitational attraction}

The action due to the gravitational attraction is represented by doubling the gravitational potential energy as shown in Eq.(10). This value also corresponds to the Schwarzschild radius as shown in Eq.(11) when the radius is put in the denominator and $\mathrm{m}$ is added in both sides of the equation. It can also be found by using the mechanical energy equation of a satellite as shown in Eq.(12).

$$
\text { Gravitational potentional energy }=G \frac{M m}{r}
$$

Action due to gravitational attraction $=2 G \frac{M m}{r}$

$$
\text { Schwarzschild radius }=r=2 G \frac{M(m)}{c^{2}(m)} \Rightarrow(m) c^{2}=2 G \frac{M(m)}{r}
$$

$$
\begin{aligned}
\text { Mechanical energy } & =\text { Kinetic Energy }- \text { Potential Energy } \\
0 & =\text { Kinetic Energy }- \text { Potential Energy } \\
\text { Potential Energy } & =\text { Kinetic Energy }
\end{aligned}
$$

$$
G \frac{M m}{r}=\frac{1}{2} m v^{2} \Rightarrow 2 G \frac{M m}{r}=m v^{2}
$$

The following equation gives the time dilation between two states by subtracting the action due to gravitational attraction.

$$
\text { Ratio }=\frac{\text { State }_{1}}{\text { State }_{2}}=\sqrt{\frac{m c^{2}-2 G \frac{M m}{r_{1}}}{m c^{2}-2 G \frac{M m}{r_{2}}}}
$$

The next equation shows the derivation to obtain the standard gravitational time dilation equation when $r_{2} \rightarrow \operatorname{Inf} f_{+}$and $r_{1}>0$.

$$
\sqrt{\frac{m c^{2}-2 G \frac{M m}{r_{1}}}{m c^{2}-0}}=\sqrt{\frac{m c^{2}}{m c^{2}}-\frac{2 G M m}{r_{1} m c^{2}}}=\sqrt{1-\frac{m}{m} \frac{2 G M}{r_{1} c^{2}}}=\sqrt{1-\frac{2 G M}{r_{1} c^{2}}}
$$


In equation Eq.(12) appears $m v^{2}$, which expresses an equivalence between the action due to motion and due to gravitational attraction. The following equivalences can also be obtained.

$$
2 G \frac{M m}{r}=G \frac{M m}{r}+\frac{1}{2} m v^{2}=m v^{2}
$$

These equivalences suggest a possible combination between Eq.(13) and Eq.(8) as explained in section 4.2 .

\section{Combination of motion and gravitational time dilation}

\subsection{Using the standard equations}

A method to calculating the relativistic effect affecting a GPS is found in [1]. This section shows how to use the standard time dilation equations to calculate the time dilation knowing the speed of the object and a gravitational mass situated next to it. The following equation can be used in which the time dilation is represented by the symbol $\gamma$.

$$
\gamma=\left(\frac{\sqrt{1-\frac{v_{1}^{2}}{c^{2}}}}{\sqrt{1-\frac{v_{2}^{2}}{c^{2}}}}\right) *\left(\frac{\sqrt{1-\frac{2 G M}{r_{1} c^{2}}}}{\sqrt{1-\frac{2 G M}{r_{2} c^{2}}}}\right)
$$

The top part represents a system in a first frame of reference. The bottom part represents the same system in another frame of reference. In the top part, $v_{1}$ represents the speed of the first system compared to a third frame of reference and the distance $r_{1}$ represents the distance from a center of mass $\mathrm{M}$. In the bottom part, $v_{2}$ represents the speed of the second system compared to the same third frame of reference and the distance $r_{2}$ represents the distance from the center of mass $\mathrm{M}$.

When $\gamma<1$, then the system represented by the top part is slower than the system represented by the bottom part. When $\gamma>1$, it means the system at the top is faster.

For example, given $v_{1}=3889, r_{1}=26378000, v_{2}=460$ and $r_{2}=6378000$, the result will be 1.000000000444252 . The values $v_{1}$ and $r_{1}$ could correspond to a GPS satellite's clock around the earth and $v_{2}$ and $r_{2}$ could correspond to a clock on the ground at rest. The satellite's clock will go faster of about 38 microseconds/day [1, 9]. When $1.000000000444252 s$ will have elapsed on the satellite's clock, only $1 s$ will have elapsed on the ground's clock.

$$
\gamma=\frac{\sqrt{1-\frac{v_{1}^{2}}{c^{2}}}}{\sqrt{1-\frac{v_{2}^{2}}{c^{2}}}} * \frac{\sqrt{1-\frac{2 G M}{r_{1} c^{2}}}}{\sqrt{1-\frac{2 G M}{r_{2} c^{2}}}}=\frac{1.000000000444252}{1}
$$

$$
\begin{aligned}
86400 \text { seconds } / \text { day } *(1.000000000444252-1) & =0.000038383 \text { seconds } / \text { day } \\
& =+38 \text { microseconds } / \text { day }
\end{aligned}
$$




\subsection{Using the energy ratio equation}

As suggested by the equivalence in Eq.(12) and Eq.(15), an equivalence between the action of motion and the action of gravitational attraction is suggested and a combination to calculate the time dilation due to motion and due to gravitational attraction is presented in the following equation in which the action due to motion and due to gravitational attraction are substracted from the value $m c^{2}$.

$$
\text { Time dilation }=\frac{\text { State }_{1}}{\text { State }_{2}}=\sqrt{\frac{m c^{2}-m v_{1}^{2}-2 G \frac{M m}{r_{1}}}{m c^{2}-m v_{2}^{2}-2 G \frac{M m}{r_{2}}}}=\sqrt{\frac{c^{2}-v_{1}^{2}-2 G \frac{M}{r_{1}}}{c^{2}-v_{2}^{2}-2 G \frac{M}{r_{2}}}}
$$

To demonstrate this possibility, a comparison is made with the result found in section 4.1 when calculating the relativistic effect affecting a GPS. Using Eq.(18), the same value is obtained.

$$
\begin{aligned}
\text { Time dilation } & =\sqrt{\frac{c^{2}-(3889 \mathrm{~m} / \mathrm{s})^{2}-2 G \frac{M}{26378000 \mathrm{~m}}}{c^{2}-(460 \mathrm{~m} / \mathrm{s})^{2}-2 G \frac{M}{6378000 \mathrm{~m}}}}=1.000000000444252 \\
\text { Dilation per day } & =86400 *(1.000000000444252-1)=0.000038383 \mathrm{~s} \\
& \approx+38 \text { microseconds }
\end{aligned}
$$

\section{Conclusion}

A time dilation equation expressed as an energy ratio was provided. This equation expresses time dilation as a comparison between two energy states. It can be used to obtain the same result as the standard time dilation equations. A demonstration was made to show a possible similitude between the value $m v^{2}$ and the work done by a drag force. A gravitational attraction energy value of $2 G M m / r$ was also introduced. An equivalence between the two values emerged, leading to a combination of both values into the energy ratio equation in order to calculate the time dilation due to motion and due to gravitational attraction.

The purpose of presenting an energy ratio equation to calculate time dilation is not to replace the standard time dilation equations that are well established and used, but to approach the principle of time dilation by using the concept of energy. This concept may assist non-experts or other persons coming from other fields of science to better understand time dilation. A point of view using energy might be for some people a more intuitive and accessible way than the geometrical transformations currently used to explain and demonstrate time dilation.

\section{Appendix A. Supplementary data}

The script to calculate the relativistic effect affecting a satellite is available on the OSF repository at https://osf.io/7gy4r 


\section{References}

[1] Ashby, N., 2003. Relativity in the global positioning system. doi:10.12942/ $\operatorname{lrr}-2003-1$.

[2] Conte, A., 2019. From relativistic time dilation to psychological time perception: an approach and model, driven by the theories of relativity, to calculate the time perceived while experiencing different situations. doi:10.31234/osf .io/dp6b5.

[3] Einstein, A., 1905a. Does the inertia of a body depend upon its energy-content. Annalen der Physik .

[4] Einstein, A., 1905b. On the electrodynamics of moving bodies (Zur Elektrodynamik bewegter Körper). Annalen der Physik .

[5] Einstein, A., 1916. The Foundation of the General Theory of Relativity (Die Grundlage der allgemeinen Relativitätstheorie). Annalen der Physik doi:10.1002/ andp. 19163540702.

[6] Einstein, A., 1920. Relativity: The Special \& General Theory.

[7] Eling, C., JACOBSON, T., MATTINGLY, D., 2006. EINSTEIN-ÆTHER THEORY, in: Deserfest, WORLD SCIENTIFIC. pp. 163-179. URL: http: //www.worldscientific.com/doi/abs/10.1142/9789812774804\{_\}0012, doi:10. 1142/9789812774804_0012.

[8] Martins, A.A., Pinheiro, M.J., 2008. On the electromagnetic origin of inertia and inertial mass. International Journal of Theoretical Physics doi:10.1007/ s10773-008-9709-y, arXiv:0802.0284.

[9] Pogge, R.W., 2017. Real-World Relativity: The GPS Navigation System. URL: https://bit.1y/2XfWPYD.

[10] Schaffner, K.F., 1972. Nineteenth-Century Aether Theories. Elsevier. URL: https://linkinghub.elsevier.com/retrieve/pii/C20130023353, doi:10.1016/ C2013-0-02335-3. 